\title{
Cornell product is an ECG marker of heart failure with preserved ejection fraction
}

\author{
Eugene SJ Tan, ${ }^{01}$ Siew Pang Chan, ${ }^{2,3}$ Chang Fen $\mathrm{Xu}_{1}{ }^{4}$ Jonathan Yap, ${ }^{4}$ \\ Arthur Mark Richards, ${ }^{1,3,5}$ Lieng Hsi Ling, ${ }^{\oplus, 2}$ David Sim, ${ }^{4,6}$ Fazlur Jaufeerally, ${ }^{6,7}$ \\ Daniel Yeo, ${ }^{8}$ Seet Yoong Loh, ${ }^{8}$ Hean Yee Ong ${ }_{1}^{9}$ Kui Toh Gerard Leong, ${ }^{10} \mathrm{Tze}_{\text {Pin Ng, }}{ }^{2}$ \\ Shwe Zin Nyunt, ${ }^{2}$ Liang Feng, ${ }^{6}$ Peter Okin, ${ }^{11}$ Carolyn SP Lam, ${ }^{4,6}$ Toon Wei Lim ${ }^{1}$
}

'Department of Cardiology, National University Heart Centre Singapore, Singapore, Singapore ${ }^{2}$ Yong Loo Lin School of Medicine, National University Singapore, Singapore, Singapore ${ }^{3}$ Cardiovascular Research Institute, National University Healthy System, Singapore, Singapore

${ }^{4}$ Department of Cardiology, National Heart Centre Singapore, Singapore, Singapore ${ }^{5}$ Christchurch Heart Institute, University of Otago, Otago, New Zealand

${ }^{6}$ Department of Medicine, DukeNUS Graduate Medical School,

Singapore, Singapore ${ }^{7}$ Department of Internal Medicine, Singapre General Hospital, Singapore, Singapore ${ }^{8}$ Department of Cardiology, Tan Tock Seng Hospital, Singapore, Singapore

${ }^{9}$ Department of Cardiology, Khoo Teck Puat Hospital, Singapore, Singapore ${ }^{10}$ Department of Cardiology, Changi General Hospital, Singapore, Singapore

${ }^{11}$ Department of Cardiology, Weill Cornell Medical College, New York City, New York, USA

\section{Correspondence to}

Dr Carolyn SP Lam, National Heart Centre Singapore,

Singapore 169609, Singapore; carolyn.lam@duke-nus.edu.sg

CSL and TWL contributed equally.

Received 7 August 2018 Revised 9 March 2019 Accepted 12 March 2019

Check for updates

(c) Author(s) (or their employer(s)) 2019. No commercial re-use. See rights and permissions. Published by BMJ.

To cite: Tan ESJ, Chan SP,

$\mathrm{Xu} C \mathrm{~F}$, et al. Heart Asia

2019:11:e011108

doi:10.1136/

heartasia-2018-011108

\section{ABSTRACT}

Objective ECG markers of heart failure (HF) with preserved ejection fraction (HFpEF) are lacking. We hypothesised that the Cornell product (CP) is a risk marker of HFpEF and has prognostic utility in HFpEF. Methods $C P=[$ (amplitude of $R$ wave in $\mathrm{aVL}+$ depth of $S$ wave in $\mathrm{V3}) \times \mathrm{QRS}$ ] was measured on baseline 12-lead ECG in a prospective Asian population-based study of 606 healthy controls (aged $55 \pm 10$ years, $45 \%$ men), 221 hypertensive controls ( $62 \pm 9$ years, $58 \%$ men) and 242 $\mathrm{HFpEF}$ ( $68 \pm 12$ years, $49 \%$ men); all with $\mathrm{EF} \geq 50 \%$ and followed for 2 years for all-cause mortality and HF hospitalisations.

Results CP increased across groups from healthy controls to hypertensive controls to HFpEF, and distinguished between $\mathrm{HFpEF}$ and hypertension with an optimal cut-off of $\geq 1800 \mathrm{~mm}^{*} \mathrm{~ms}$ (sensitivity $40 \%$, specificity $85 \%$ ). Age, male sex, systolic blood pressure (SBP) and heart rate were independent predictors of $\mathrm{CP} \geq 1800 \mathrm{~mm}^{*} \mathrm{~ms}$, and $\mathrm{CP}$ was associated with echocardiographic E/e' $(r=0.27, p<0.01)$ and left ventricular mass index $(r=0.46, p<0.01)$. Adjusting for clinical and echocardiographic variables and log $\mathrm{N}$-terminal pro B-type natriuretic peptide (NT-proBNP), $\mathrm{CP} \geq 1800 \mathrm{~mm}^{*} \mathrm{~ms}$ was significantly associated with HFpEF (adjusted OR 2.7, 95\% CI 1.0 to 7.0). At 2-year follow-up, there were 29 deaths and 61 HF hospitalisations, all within the HFpEF group. Even after adjusting for log NT-proBNP, clinical and echocardiographic variables, $\mathrm{CP} \geq 1800 \mathrm{~mm}$ * $\mathrm{ms}$ remained strongly associated with a higher composite endpoint of all-cause mortality and HF hospitalisations (adjusted HR $2.1,95 \% \mathrm{Cl} 1.2$ to 3.5$)$.

Conclusion The Cornell product is an easily applicable ECG marker of HFpEF and predicts poor prognosis by reflecting the severity of diastolic dysfunction and LV hypertrophy.

\section{INTRODUCTION}

Heart failure with preserved ejection fraction (HFpEF) has been studied with great interest in recent times. In addition to increased systemic afterload, ventricular stiffness and impaired diastolic function have also been suggested in the pathophysiology of HFpEF. ${ }^{1-3}$ The assessment of HFpEF involves objective evidence of diastolic dysfunction in the presence of normal systolic function on transthoracic echocardiography. Left ventricular hypertrophy $(\mathrm{LVH})$, determined by left ventricular mass, has been widely used as a surrogate of diastolic function in the diagnosis of HFpEF. ${ }^{4-6}$

\section{Key questions}

What is already known about this subject?

- Heart failure (HF) with preserved ejection fraction (HFpEF) is associated with significant morbidity and mortality and remains a major burden to healthcare systems globally.

- Diagnosis of HFpEF requires comprehensive transthoracic echocardiograms and may be aided by adjunctive serum biomarkers such as $\mathrm{N}$-terminal pro B-type natriuretic peptide. ECG markers of HFpEF are lacking.

\section{What does this study add?}

- The ECG marker, Cornell product, at a cutoff of greater than or equal to $1800 \mathrm{~mm}^{*} \mathrm{~ms}$ distinguishes $\mathrm{HFpEF}$ from hypertension, with threefold increased odds of HFpEF. In patients with HFpEF, a Cornell product of $\geq 1800$ $\mathrm{mm}^{*} \mathrm{~ms}$ is also associated with two times higher likelihood of all-cause mortality and HF hospitalisations.

\section{How might this impact on clinical practice?}

- The ECG Cornell product is an easily derived and applicable marker of HFpEF and is associated with poorer outcomes above a cutoff of $\geq 1800 \mathrm{~mm}^{*} \mathrm{~ms}$.

A quick and simple way to screen for LVH without the use of echocardiography would be through the widely available 12-lead ECG. Several ECG voltage criteria have been developed in the assessment of LVH, including the Sokolow-Lyon and Cornell voltage criteria. ${ }^{78}$ The Cornell product (CP), ${ }^{9}$ an ECG voltage-duration product, was found to have greater sensitivity for $\mathrm{LVH}^{910}$ and has been shown to be associated with diastolic dysfunction. ${ }^{11}$

We hypothesised that $\mathrm{CP}$ would be an alternate way of identifying patients at high risk of $\mathrm{HFpEF}$ and sought to determine the implications of $\mathrm{CP}$ in patients with HFpEF.

\section{METHODS \\ Study population}

The study population included participants from the Singapore Heart Failure Outcomes and Phenotypes (SHOP) Study, ${ }^{12}$ a prospective study of consecutive in-patients and outpatients identified with heart failure (HF) from six centres in Singapore followed up over 2 years, as well as healthy 
controls without HF from the community-based Singapore Longitudinal Ageing Study (SLAS). ${ }^{13}$ Participants in SLAS were adults randomly sampled from the community via door-to-door census of residents in contiguous precincts within five districts in Southeastern Singapore. All participants underwent detailed clinical profiling with prospective 12-lead resting ECG, comprehensive Doppler echocardiography, phlebotomy for biomarker measurements and were followed up for all-cause death and HF hospitalisations. In compliance with the Declaration of Helsinki, informed consent was obtained from all participants. As this was a prospective study, there was no recall bias and only participants with HF were recruited in the HFpEF group, hence avoiding selection bias. There were minimal missing data, and hence imputation was not performed. Loss to follow-up was minimal at $<1 \%$.

In this study with 2087 subjects (1100 with HF), participants with reduced $\mathrm{EF}<50 \%$ or left bundle branch block were excluded. The remaining 1069 participants were split into three groups (827 participants from SLAS in both groups I and II and 242 participants from SHOP in group III).

- Group I: healthy controls (no hypertension and no HF).

- Group II: participants with hypertension only without HF.

- Group III: participants with HFpEF.

Importantly, the diagnosis of HFpEF was based on the validated clinical diagnosis of HF (the presence of typical symptoms and signs, established by cardiologists and validated by investigators) and the presence of LVEF $\geq 50 \%$ (independently verified in an echocardiography core laboratory). As previously published, ${ }^{14} 49$ out of $50 \mathrm{HFpEF}$ cases in a substudy satisfied the detailed 2007 European diagnostic criteria for $\mathrm{HFpEF}^{4}$

\section{Electrocardiography}

Resting baseline standard 12-lead ECGs were done at recruitment. ECGs were performed in a standardised fashion across all participating centres. To minimise intercentre and interobserver variability, the same ECG machine (Mortara ELI 250, Milwaukee, Wisconsin, USA) was used across all centres and ECGs calibrated at $25 \mathrm{~mm} / \mathrm{s}$ and $1 \mathrm{mV} / \mathrm{cm}$ were read by a single, independent, trained reader. ECG variables of interest including $\mathrm{R}$ wave amplitude in aVL (RaVL), S wave depth in V3 (SV3) and QRS duration were recorded. ECG CP was defined as: $\mathrm{CP}=\left((\mathrm{RaVL}+\mathrm{SV} 3)^{*} \mathrm{QRS}\right.$ duration $) .{ }^{9}$

\section{Echocardiography}

Comprehensive two-dimensional echocardiograms, including M-mode, pulse wave Doppler and tissue Doppler imaging, were performed by experienced sonographers. Commercially available ultrasound systems (Vivid seven and E9, General Electric, Milwaukee, Wisconsin, USA) equipped with broadband transducers were used across all centres. Measurements of ejection fraction, mitral inflow E and A velocities, septal and LV lateral annular early diastolic velocities (e'), LV dimensions including interventricular septum thickness, LV internal diameter and inferolateral wall thickness were made by trained echocardiographers blinded to ECG data. As all patients included in this study had preserved LVEF, average E/e' $>14$ was used as the definitive marker of diastolic dysfunction in our study as per American Society of Echocardiography recommendations. ${ }^{15} \mathrm{LV}$ mass was calculated using the Cube formula ${ }^{16}$ and LV mass index (LVMI) obtained by indexing LV mass to body surface area.

\section{Statistical analysis}

Baseline characteristics were reported as percentages (\%) for categorical variables and mean $\pm S D$ or median with IQR for continuous variables. Bivariate analyses were carried out with the $\chi^{2}$ (categorical) or Kruskal-Wallis test (continuous). Pairwise correlations were made between $\mathrm{CP}$ and echocardiographic markers of LVMI and E/e' ratio. An optimal CP cut-off to distinguish between HFpEF and hypertension without HF was identified using receiver operating characteristics (ROC) curves. Kaplan-Meier curves were generated to compare time to all-cause mortality, time to first recurrent HF hospitalisation and time to composite event (all-cause mortality and first recurrent HF hospitalisation) according to the optimal CP cut-off.

To ascertain the association of $\mathrm{CP}$ with clinical covariates and its prognostic significance at the identified cut-off, a generalised structural equation model (gSEM) was constructed. ${ }^{17}$ The association between $\mathrm{CP}$ and clinical and echocardiographic variables (age, gender, ethnicity, blood pressure, heart rate, E/e' and LVMI) were determined, and time to events (all-cause mortality, first recurrent HF hospitalisation and composite event) was estimated in patients with HFpEF only. Two-sided statistical tests were performed at $5 \%$ level of significance. All data analyses were carried out with Stata MP V.14.

\section{RESULTS}

\section{Baseline characteristics}

Baseline characteristics of the study population by group are shown in table 1. A total of 1069 adults (median CP 1065 $\mathrm{mm}$ *ms (IQR 736-1517)) were included in this study, with 606 healthy controls $(55 \pm 10$ years, $45 \%$ men), 221 controls with hypertension but without HF (62 \pm 9 years, $58 \%$ men) and 242 with HFpEF (68 \pm 12 years, $49 \%$ men). In the HFpEF group, $86 \%$ of participants had hypertension. Median CP increased across groups from $924 \mathrm{~mm} * \mathrm{~ms}$ in healthy controls to 1210 $\mathrm{mm} * \mathrm{~ms}$ in hypertension without HF to $1523 \mathrm{~mm} * \mathrm{~ms}$ in HFpEF $(\mathrm{p}<0.001)$ (figure 1). Similarly, there was an increasing trend in age, body mass index, QRS duration (ms), $\mathrm{R}$ wave amplitude in aVL, depth of S wave in V3, E/e', LVMI and N-terminal pro B-type natriuretic peptide (NT-proBNP) across all three groups (table 1).

The optimal CP cut-off for predicting HFpEF from hypertension without HF (Group II and III only) was identified to be greater than or equal to $1800 \mathrm{~mm} * \mathrm{~ms}$, with sensitivity and specificity of $40 \%$ and $85 \%$, respectively (area under ROC curve $0.62 ; 95 \%$ CI 0.56 to 0.67 ). Sex-specific cut-offs were also tested but they did not significantly improve diagnostic accuracy. Age, gender, SBP and heart rate were significant independent predictors of a CP $\geq 1800 \mathrm{~mm}^{*} \mathrm{~ms}$ (table 2). The associations between the optimal CP cut-off and ethnicity were not significant when compared with the reference Chinese group (table 2).

\section{Association of CP with HFpEF}

$\mathrm{CP} \geq 1800 \mathrm{~mm}$ "ms was significantly associated with sixfold increased odds of HFpEF after adjusting for clinical variables of age, sex, ethnicity, SBP and heart rate (table 3) among patients from Groups II and III. When adjusted for clinical variables and echocardiographic variables of LVMI and mitral E/e', CP $\geq 1800$ $\mathrm{mm}$ * ms was associated with twofold increased odds of HFpEF (table 3). CP correlated positively with NT-proBNP, which was also a significant predictor of HFpEF (adjusted OR (AOR) of $\operatorname{logNT}$-proBNP 6.03, 95\% CI 3.76 to 9.69) ( $r=0.27, \mathrm{p}<0.001$ ). However, after adjusting for both clinical and echocardiographic variables, as well as logNT-proBNP, CP remained strongly predictive of HFpEF with threefold increased odds (table 3). Even when analysed as a continuous variable, CP remained 


\begin{tabular}{|c|c|c|c|c|}
\hline & Control & HYPT & HFpEF & $P$ value \\
\hline$N$ & 606 & 221 & 242 & \\
\hline Age, years & $55.2 \pm 10.0$ & $62.4 \pm 8.8$ & $68.2 \pm 11.5$ & $<0.001$ \\
\hline Sex, male (\%) & $270(45)$ & $128(58)$ & $118(49)$ & 0.003 \\
\hline \multicolumn{5}{|l|}{ Ethnicity } \\
\hline Chinese & $418(69)$ & $181(82)$ & $151(62)$ & 0.001 \\
\hline Malay & $121(20)$ & $29(13)$ & $67(28)$ & \\
\hline Indian & $41(7)$ & $10(5)$ & $21(9)$ & \\
\hline Height, m & $1.61 \pm 0.09$ & $1.61 \pm 0.08$ & $1.58 \pm 0.08$ & 0.001 \\
\hline Weight, kg & $62.9 \pm 12.3$ & $66.2 \pm 11.4$ & $69.8 \pm 15.7$ & $<0.001$ \\
\hline BMI, $\mathrm{kg} / \mathrm{m}^{2}$ & $24.3 \pm 4.0$ & $25.7 \pm 3.6$ & $27.9 \pm 5.9$ & $<0.001$ \\
\hline $\mathrm{SBP}, \mathrm{mm} \mathrm{Hg}$ & $125.9 \pm 17.1$ & $142.7 \pm 16.5$ & $133.2 \pm 21.8$ & $<0.001$ \\
\hline DBP, mm Hg & $73.7 \pm 10.7$ & $81.1 \pm 10.2$ & $70.7 \pm 12.3$ & $<0.001$ \\
\hline $\begin{array}{l}\text { Hear rate, beats } \\
\text { per minute }\end{array}$ & $67.3 \pm 9.8$ & $66.6 \pm 9.8$ & $72.4 \pm 12.8$ & $<0.001$ \\
\hline $\mathrm{RaVL}, \mathrm{mm}$ & $3.7 \pm 2.8$ & $5.3 \pm 3.0$ & $6.6 \pm 4.3$ & $<0.001$ \\
\hline $\mathrm{SV} 3, \mathrm{~mm}$ & $7.5 \pm 4.0$ & $9.2 \pm 4.4$ & $10.7 \pm 6.7$ & $<0.001$ \\
\hline $\begin{array}{l}\text { QRS duration, } \\
\mathrm{ms}\end{array}$ & $85.4 \pm 11.4$ & $88.0 \pm 12.1$ & $93.6 \pm 15.3$ & $<0.001$ \\
\hline $\begin{array}{l}\text { Cornell } \\
\text { product*, } \\
\mathrm{mm}^{*} \mathrm{~ms}\end{array}$ & $924(624,1248)$ & $1210(913,1633)$ & $1523(979,2126)$ & $<0.001$ \\
\hline E/e' ratio & $8.1 \pm 2.3$ & $9.6 \pm 2.9$ & $14.5 \pm 7.4$ & $<0.001$ \\
\hline LVMI, $g / \mathrm{m}^{2}$ & $77.3 \pm 18.2$ & $90.3 \pm 19.9$ & $113.4 \pm 31.0$ & $<0.001$ \\
\hline NT-proBNP*, & $50(28,81)$ & $60(32,101)$ & $1026(345,2385)$ & $<0.001$ \\
\hline
\end{tabular}

$\mathrm{pg} / \mathrm{mL}$

\begin{tabular}{lllcc}
$\begin{array}{l}\text { All-cause } \\
\text { deaths }\end{array}$ & 0 & 0 & $29(13)$ & NA \\
$\begin{array}{l}\text { First HF } \\
\begin{array}{l}\text { admission on } \\
\text { enrolment }\end{array}\end{array}$ & 0 & 0 & $61(25)$ & NA \\
\hline
\end{tabular}

Differences in baseline characteristics across all three groups studied.

${ }^{*}$ Expressed as median (IQR), all other values expressed as average \pm SD.

$B M I$, body mass index; DBP, diastolic blood pressure; E/e', Average mitral annular $E$ velocity to e' ratio; $H F$, heart failure; $H F p E F$, $H F$ with preserved ejection fraction; $H R$, hazard ratio; HYPT, hypertension without heart failure; LVMI, left ventricular mass index; NA, Not available; NT-proBNP, N-terminal pro B-type natriuretic peptide; RaVL, amplitude of $\mathrm{R}$ wave in $\mathrm{aVL}$; $\mathrm{SBP}$, systolic blood pressure; SV3, depth of $\mathrm{S}$ wave in V3.

strongly predictive with $10 \%$ increased odds of HFpEF for every $100 \mathrm{~mm} * \mathrm{~ms}$ increase in CP after adjusting for clinical variables.

CP was also significantly associated with the echocardiographic markers of diastolic dysfunction, $E / e^{\prime} \quad(r=0.27$, $\mathrm{p}<0.001)$ and LVMI $(\mathrm{r}=0.46, \mathrm{p}<0.01)$. Stratifying by gender,

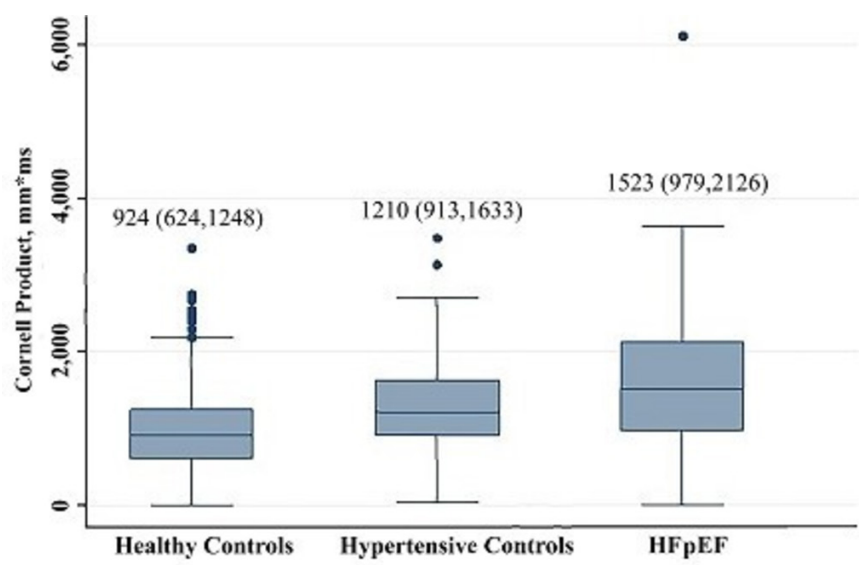

Median (interquartile range) of Cornell Product across the three study groups depicted. Dots represent outliers.

HFpEF: heart failure with preserved ejection fraction

Figure 1 Distribution of Cornell product across study population.
Table 2 Association of clinical covariates with $\mathrm{CP} \geq 1800 \mathrm{~mm}^{*} \mathrm{~ms}$

\begin{tabular}{|c|c|c|c|}
\hline Clinical covariates & $\begin{array}{l}\text { Adjusted } \\
\mathrm{OR}\end{array}$ & $95 \% \mathrm{Cl}$ & $\begin{array}{l}P \\
\text { value }\end{array}$ \\
\hline Age per 10 years & 1.46 & 1.24 to 1.73 & $<0.001$ \\
\hline Sex (female) & 0.40 & 0.27 to 0.58 & $<0.001$ \\
\hline \multicolumn{4}{|l|}{ Ethnicity } \\
\hline Chinese & Reference & & \\
\hline Malay & 0.91 & 0.55 to 1.51 & 0.720 \\
\hline Indian & 0.93 & 0.44 to 1.98 & 0.851 \\
\hline SBP per $10 \mathrm{~mm} \mathrm{Hg}$ & 1.14 & 1.05 to 1.26 & 0.003 \\
\hline $\begin{array}{l}\text { Heart rate per } 10 \text { beats per } \\
\text { minute }\end{array}$ & 1.26 & 1.08 to 1.49 & 0.004 \\
\hline
\end{tabular}

Multivariate analysis of clinical variables in association with $\mathrm{CP} \geq 1800 \mathrm{~mm}{ }^{*} \mathrm{~ms}$, with adjusted $\mathrm{OR}$ expressed for age, SBP and heart rate in per 10 units increase. CP, Cornell product; SBP, systolic blood pressure.

CP maintained its correlation with LVMI $(r=0.42, p<0.001$ in males and $\mathrm{r}=0.43, \mathrm{p}<0.001$ in females).

\section{Association of $\mathrm{CP}$ with outcomes}

During follow-up of 2 years, there were 29 deaths and 61 first HF admissions from enrolment, all of which occurred in the HFpEF group. The relationships of CP $\geq 1800 \mathrm{~mm} * \mathrm{~ms}$ to time to events are depicted in table 4. In patients with HFpEF (Group III only), those with $\mathrm{CP} \geq 1800 \mathrm{~mm}$ *ms were associated with a twofold increased risk of earlier composite outcomes of all-cause death and recurrent HF hospitalisation after adjustment for age, sex, ethnicity, SBP, heart rate, E/e', LVMI as well as logNTproBNP (table 4 , figure 2 ). There was no significant interaction between $\operatorname{logNT}$-proBNP and CP $1800\left(\mathrm{p}_{\text {interaction }}=0.81\right)$ on their association with outcomes. The increased association was noted when all-cause death and HF hospitalisation were accessed individually (table 4).

\section{DISCUSSION}

We demonstrate that the CP is an important risk ECG marker of HFpEF, and at a cut-off greater or equal to $1800 \mathrm{~mm} * \mathrm{~ms}, \mathrm{CP}$ is a strong marker of HFpEF and poorer prognosis. Moreover, its prognostic value is independent of NT-proBNP and age.

\section{Association of $\mathrm{CP}$ and clinical covariates}

The CP was developed as an extension of the ECG Cornell voltage criteria for better sensitivity in detecting LVH. ${ }^{9}{ }^{10}$ Casale et al found significant sex differences in SV3, while age did not influence ECG detection of $\mathrm{LVH}^{7}{ }^{7}$ Similarly, we found that females were less likely to have a CP $\geq 1800 \mathrm{~mm}$ *ms than men, which may be accounted for by their lower LV mass. Apart from

Table 3 Association of $\mathrm{CP} \geq 1800 \mathrm{~mm}^{*} \mathrm{~ms}$ with $\mathrm{HFpEF}$

\begin{tabular}{lllc}
\hline Adjusting variables & $\begin{array}{l}\text { Adjusted } \\
\text { OR }\end{array}$ & $95 \% \mathrm{Cl}$ & P value \\
\hline $\begin{array}{l}\text { Clinical variables } \\
\begin{array}{l}\text { Clinical and echocardiographic } \\
\text { variables }\end{array}\end{array}$ & 6.00 & 3.38 to 10.51 & $<0.001$ \\
$\begin{array}{l}\text { Clinical, echocardiographic variables } \\
\text { and logNT-proBNP }\end{array}$ & 2.69 & 1.13 to 4.61 & 0.02 \\
\hline
\end{tabular}

Multivariate adjustment of $\mathrm{CP} \geq 1800 \mathrm{~mm}{ }^{*} \mathrm{~ms}$ with clinical and/or echocardiographic variables.

Clinical variables: age, sex, SBP and heart rate.

Echocardiographic variables: mitral E/e' ratio and LVMI

$\mathrm{CP}$, Cornell product; $\mathrm{HFpEF}$, heart failure with preserved ejection fraction; LVMI, left ventricular mass index; NT-proBNP, N-terminal pro B-type natriuretic peptide; SBP, systolic blood pressure. 
Table 4 Association of $\mathrm{CP} \geq 1800 \mathrm{~mm}^{*} \mathrm{~ms}$ with time to events in patients with heart failure with preserved ejection fraction

\begin{tabular}{|c|c|c|c|}
\hline & HR & $95 \% \mathrm{Cl}$ & $\begin{array}{l}P \\
\text { value }\end{array}$ \\
\hline \multicolumn{4}{|c|}{ Time to all-cause death } \\
\hline Unadjusted & 2.67 & 1.23 to 5.79 & 0.013 \\
\hline Adjusted & 2.91 & 1.14 to 7.42 & 0.026 \\
\hline \multicolumn{4}{|c|}{ Time to first heart failure hospitalisation } \\
\hline Unadjusted & 1.95 & 1.16 to 3.27 & 0.012 \\
\hline Adjusted & 1.97 & 1.04 to 3.68 & 0.037 \\
\hline \multicolumn{4}{|c|}{ Time to composite* } \\
\hline Unadjusted & 2.08 & 1.33 to 3.25 & 0.001 \\
\hline Adjusted & 2.06 & 1.21 to 3.51 & 0.008 \\
\hline \multicolumn{4}{|c|}{$\begin{array}{l}\text { Multivariate adjustment of } \mathrm{CP} \geq 1800 \mathrm{~mm}{ }^{*} \mathrm{~ms} \text { with clinical, echocardiographic and NT- } \\
\text { proBNP for various outcomes. } \\
\text { Adjusted models: adjusted for age per } 10 \text { years, sex, ethnicity, systolic blood pressure per } \\
10 \mathrm{~mm} \mathrm{Hg} \text {, heart rate per } 10 \text { beats per minute, logNT-proBNP, E/e' and left ventricular } \\
\text { mass index. } \\
\text { *Composite of all-cause death and heart failure hospitalisation. } \\
\mathrm{CP} \text {, Cornell product; E/e', Average mitral annular E velocity to e' ratio; NT-proBNP, } \\
\text { N-terminal pro B-type natriuretic peptide. }\end{array}$} \\
\hline
\end{tabular}

gender, we observed that age, heart rate and SBP were independently associated with CP. The association of ECG parameters with age, sex and ethnicity has been previously described, with men having wider QRS and deeper SV3, while age was associated with wider QRS and taller RaVL and Malays with wider QRS duration. ${ }^{18}$ We observed no inter-ethnic differences in CP, likely due to the small number of Malays and Indians as compared with Chinese. The effect of heart rate on $\mathrm{CP}$ is more uncertain, although recent studies have shown reductions in
QRS duration with increased heart rates. ${ }^{19}{ }^{20}$ The effect of heart rate on RaVL and SV3 remains unknown.

\section{Association of CP and HFpEF}

Even though Molloy et al ${ }^{10}$ had previously found sex-specific values for ECG Cornell voltage criteria, we did not find significant differences in the association between $\mathrm{CP}$ and HFpEF when sex-specific cut-offs were used. Sex-specific cut-offs in the association between $\mathrm{CP}$ and HFpEF were therefore not applied, and a cut-off of $C P \geq 1800 \mathrm{~mm} * \mathrm{~ms}$ was used in both sexes.

The CP has higher sensitivity in patients with more severe $\mathrm{LVH} .{ }^{10}$ Okin et al showed that above a median LVMI of $153 \mathrm{~g} /$ $\mathrm{m}^{2}$, CP had a sensitivity of $41 \%$ compared with $33 \%$ in LVMI $<153 \mathrm{~g} / \mathrm{m}^{2}$. ${ }^{9}$ Our study reiterates these findings, showing an increase in $\mathrm{CP}$ across the three groups from healthy controls to hypertension to HFpEF, corresponding with higher LVMI in the same rank order. The moderate to strong correlation with LVMI indicates a direct influence of LVH on the constituent ECG components of $\mathrm{CP}$, arising from prolonged impulse conduction in hypertrophied myocardium and velocity changes secondary to intramural fibrosis. ${ }^{1021}$ Previous studies have found a diagnostic threshold for LVH at CP of $2440 \mathrm{~mm} * \mathrm{~ms}^{910}$ and $2370 \mathrm{~mm} * \mathrm{~ms}$ more recently in a Japanese study. ${ }^{22}$ Median CP levels in patients with HFpEF from our study were however lower, suggesting a risk of $\mathrm{HF}$ at even lower $\mathrm{CP}$ levels.

Krepp et al demonstrated that CP was a strong predictor of diastolic dysfunction, with fivefold increased odds when CP was $\geq 1595 \mathrm{~mm}$ * ms. ${ }^{11}$ Although our study was not intended to determine the relationship between $\mathrm{CP}$ and diastolic dysfunction, the correlation between $\mathrm{CP}$ and $\mathrm{E} / \mathrm{e}^{\prime}(\mathrm{r}=0.27, \mathrm{p}<0.001)$ and trend towards HF with higher CP values supports such an
A

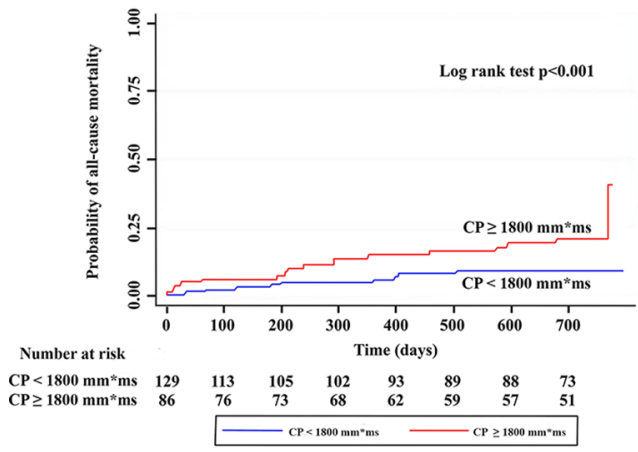

B

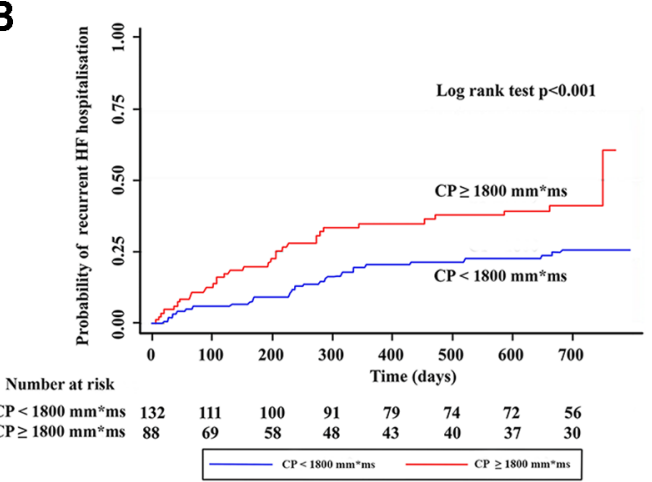

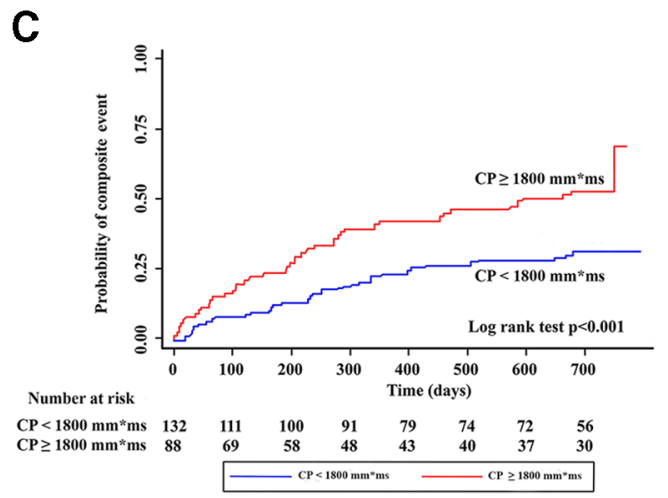

Figure 2 (A) $C P \geq 1800 \mathrm{~mm}^{*} \mathrm{~ms}$ and all-cause mortality in HFpEF. (B) $\mathrm{CP} \geq 1800 \mathrm{~mm}^{*} \mathrm{~ms}$ and recurrent HF hospitalisation in HFpEF. (C) CP $\geq 1800$ $\mathrm{mm}^{*} \mathrm{~ms}$ and composite of all-cause mortality and recurrent $\mathrm{HF}$ hospitalisation. (A-C) Kaplan-Meier curves of the association of $\mathrm{CP}$ at the optimal cutoff of $1800 \mathrm{~mm} *$ ms with composite of all-cause mortality and first recurrent $\mathrm{HF}$ hospitalisation in patients with HFpEF. CP, Cornell product; HF, heart failure; $\mathrm{HFpEF}$, HF with preserved ejection fraction. 
association. Our patients with $\mathrm{HFpEF}$ had a mean $\mathrm{E} / \mathrm{e}^{\prime}$ ratio of 14.5 and a corresponding median CP of $1523 \mathrm{~mm} * \mathrm{~ms}$, suggesting that even at lower CP values $1595 \mathrm{~mm} * \mathrm{~ms}$, patients may already have diastolic dysfunction and be at risk of progression to HF. In contrast, hypertensive participants without HF had a mean E/e' of 9.6 and a corresponding median CP of 1210 $\mathrm{mm} * \mathrm{~ms}$.

Results from the Dallas Heart Study showed that compared with ECG alone, NT-proBNP had higher sensitivity and lower specificity for LVH, but the combination of NT-proBNP and ECG LVH criteria significantly improved the discrimination of $\mathrm{LVH}^{23}$ The positive correlation of NT-proBNP with CP and their independent associations with $\mathrm{HFpEF}$ further demonstrate that both are complementary markers in screening for $\mathrm{LVH}^{23}$ Even after accounting for NT-proBNP, CP at the cut-off value of greater than or equal to $1800 \mathrm{~mm} * \mathrm{~ms}$ was still predictive of HFpEF from hypertensive heart disease without HF.

The association of CP with HFpEF is likely a reflection of the severity of LVH as well as diastolic dysfunction. Although HFpEF remains a clinical diagnosis requiring history, physical examination and echocardiographic findings, ECGs are widely available, affordable and commonly used to screen symptomatic patients. In this context, the recognition of $\mathrm{CP}$ as a marker of increased risk of HFpEF may be useful.

\section{Prognostic utility of CP}

Data on the prognostic utility of CP in HFpEF are scarce. The associations of $\mathrm{CP}$ with death and $\mathrm{HF}$ rehospitalisations in chronic HF was demonstrated by Otaki et al, ${ }^{22}$ and the incremental value of $\mathrm{CP}$ in predicting $\mathrm{HF}$ when combined with echocardiographic LVH criteria by Gerdts et al ${ }^{24}$ However, both studies included patients with reduced LVEF. Other studies have also shown that $\mathrm{CP}$ is a useful predictor of adverse cardiac events and stroke ${ }^{25} 26$ but did not take LVEF into account. However, in our study of only patients with LVEF $\geq 50 \%$, we showed that in addition to predicting increased HFpEF hospitalisations, CP $\geq 1800$ also predicts for earlier HF hospitalisations.

The association of CP with mortality had previously been established by Sundström et al. At the LVH diagnostic threshold of $2440 \mathrm{~mm} * \mathrm{~ms}$, CP conferred HRs of 3.56 and 3.82 for cardiovascular and total mortality, respectively, after adjusting for cardiovascular risk factors. ${ }^{27}$ The Losartan Intervention for Endpoint reduction in hypertension study demonstrated a reduction in $\mathrm{HF}$ hospitalisations ${ }^{28}$ and lower risks of cardiovascular mortality, myocardial infarctions and stroke with $\mathrm{CP}$ regression during antihypertensive therapy. ${ }^{29}$ Our study extends these findings to patients with $\mathrm{HFpEF}$, some of whom were not hypertensive. We report the ability of $\mathrm{CP} \geq 1800 \mathrm{~mm}$ *ms to predict worse outcomes in these patients, which was independent of multiple factors known to impact outcomes. Thus, patients may already be at higher risk of HF and mortality, even before florid expression of ECG LVH. ${ }^{27}$

BNPs have been shown to predict worse outcomes in HFpEF and $\mathrm{HFrEF}$ - for a given BNP level, the risk of all-cause mortality and HF hospitalisation is similar in both HFpEF and HFrEF. ${ }^{30}$ In our study, NT-proBNP was similarly a significant predictor of HF readmissions and all-cause mortality. However, despite the presence of NT-proBNP as a strong predictor of poorer outcomes, CP $\geq 1800 \mathrm{~mm}$ * ms remained independently associated with increased composite events. Our study suggests that in addition to BNP or NT-proBNP, CP may be an inexpensive and readily available prognostic marker among patients with HFpEF.

\section{Limitations}

The small number of mortality events limited the survival analyses and reflected brief follow-up. We analysed $\mathrm{CP}$ at a single time point but acknowledge that time trends of $\mathrm{CP}$ and their relationship to outcomes would be of interest. Participants in our study were all Asians, in particular, a predominantly ethnic Chinese population. Given ethnic variations in the prevalence of hypertension and ECG parameters, this may potentially limit the generalisability of our findings, in particular the cut-off point of greater than or equal to $1800 \mathrm{~mm}^{*} \mathrm{~ms}$ across all populations. Differences between body and heart size, as well as ECG differences between Caucasian and Asian populations are well recognised, and comparative future studies are warranted.

\section{CONCLUSION}

The ECG CP is an important risk marker of HFpEF and is independently related to poorer outcomes in HFpEF. It is a useful and easily measured alternative to identify patients at high risk of HFpEF.

Acknowledgements The authors thank the sonographers, technicians, nurses and staff at the Cardiovascular Research Institute, National University Heart Centre, Singapore, for their technical and clinical support.

Contributors ET and SPC contributed to data analysis and writing of the manuscript. CFX, JY, AMR, LHL, DS, FJ, DY, SYL, HYO, KTGL, TPN, SZN and LF contributed to the conduction of the study. CFX, SZN and LF contributed to the data acquisition. ET, JY, AMR, LHL, DS, FJ, DY, SYL, HYO, KTGL and TPN contributed to the reporting. ET, $A M R, P O, C L$ and TWL contributed to the planning. ET, SPC, AMR, PO, CL and TWI contributed to the interpretation of data. CFX, JY, AMR, LHL, DS, FJ, DY, SYL, HYO, KTGL, TPN, SZN, LF, PO, CL and TWL contributed to the review of the manuscript.

Funding This work (SHOP study) was supported by the National Medical Research Council, Singapore (grant no. R-172-003-219-511); the A*STAR-NZ HRC (grant no. JGC 10_027); and the Clinician Scientist Award (CL) to CL, and Singapore Longitudinal Aging Study supported by National Medical Research Council, Singapore (NMRC/CIRG/1348/2012)

; National Medical Research Council (CIRG14may017); and Ministry of Health, Health Services Research - (HSRG0016/2010) to TPN The study sponsors had no role in the design, collection, analysis, interpretation of data, writing of report and decision to submit for publication.

Competing interests $C L$ reports grants from National Medical Research Council of Singapore, non-financial support from Boston Scientific, non-financial support from Bayer, non-financial support from Thermofisher, non-financial support from Medtronic, non-financial support from Vifor Pharma, other from Bayer, other from Novartis, other from Takeda, other from Merck, other from Astra Zeneca, other from Janssen Research and Development, other from LLC, other from Menarini, other from Boehringer Ingelheim, other from Abbott Diagnostics, other from Corvia, other from Stealth BioTherapeutics, other from Roche, other from Amgen, outside the submitted work. In addition, CL has a patent PCT/SG2016/050217 pending. CL reports grants from National Medical Research Council, Singapore (grant no. R-172003-219-511), grants from A*STAR-NZ HRC (grant no. JGC 10_027), grants from Clinician Scientist Award (CL) to CL, TPN reports grants from Singapore Longitudinal Aging Study supported by National Medical Research Council, Singapore (NMRC/ CIRG/1348/2012)

\section{Patient consent for publication Not required.}

Ethics approval Ethics approval was obtained from the National Healthcare Group Domain Specific Review Board (reference number 2010/00114) and Singhealth Centralised Institutional Review Board (reference number 2010/196/C). Informed consent was obtained from all patients prior to participation.

Provenance and peer review Not commissioned; externally peer reviewed.

Author note All the co-authors listed in this manuscript fulfill criteria of authorship. 


\section{REFERENCES}

1. Lam CSP, Roger VL, Rodeheffer RJ, et al. Cardiac structure and ventricular-vascular function in persons with heart failure and preserved ejection fraction from Olmsted County, Minnesota. Circulation 2007;115:1982-90.

2. Melenovsky V, Borlaug BA, Rosen B, et al. Cardiovascular features of heart failure with preserved ejection fraction versus nonfailing hypertensive left ventricular hypertrophy in the urban Baltimore community: the role of atrial remodeling/dysfunction. J Am Coll Cardiol 2007;49:198-207.

3. Paulus WJ, Tschöpe C. A novel paradigm for heart failure with preserved ejection fraction: comorbidities drive myocardial dysfunction and remodeling through coronary microvascular endothelial inflammation. J Am Coll Cardiol 2013;62:263-71.

4. Paulus WJ, Tschöpe C, Sanderson JE, et al. How to diagnose diastolic heart failure: a consensus statement on the diagnosis of heart failure with normal left ventricular ejection fraction by the heart failure and echocardiography associations of the European Society of cardiology. Eur Heart J 2007;28:2539-50.

5. Vasan RS, Levy D. Defining diastolic heart failure: a call for standardized diagnostic criteria. Circulation 2000;101:2118-21.

6. Yturralde RF, Gaasch WH. Diagnostic criteria for diastolic heart failure. Prog Cardiovasc Dis 2005;47:314-9.

7. Casale PN, Devereux RB, Kligfield P, et al. Electrocardiographic detection of left ventricular hypertrophy: development and prospective validation of improved criteria. J Am Coll Cardiol 1985;6:572-80.

8. Sokolow M, Lyon TP. The ventricular complex in left ventricular hypertrophy as obtained by unipolar precordial and limb leads. Am Heart J 1949;37:161-86.

9. Okin PM, Roman MJ, Devereux RB, et al. Electrocardiographic identification of increased left ventricular mass by simple voltage-duration products. J Am Coll Cardiol 1995;25:417-23.

10. Molloy TJ, Okin PM, Devereux RB, et al. Electrocardiographic detection of left ventricular hypertrophy by the simple QRS voltage-duration product. J Am Coll Cardiol 1992;20:1180-6.

11. Krepp JM, Lin F, Min JK, et al. Relationship of electrocardiographic left ventricular hypertrophy to the presence of diastolic dysfunction. Ann Noninvasive Electrocardiol 2014;19:552-60.

12. Santhanakrishnan R, Ng TP, Cameron VA, et al. The Singapore heart failure outcomes and phenotypes (shop) study and prospective evaluation of outcome in patients with heart failure with preserved left ventricular ejection fraction (people) study: rationale and design. J Card Fail 2013;19:156-62.

13. Tan $\mathrm{CSH}$, Chan YH, Wong TY, et al. Prevalence and risk factors for refractive errors and ocular biometry parameters in an elderly Asian population: the Singapore longitudinal aging study (SLAS). Eye 2011;25:1294-301.

14. Santhanakrishnan R, Chong JPC, Ng TP, et al. Growth differentiation factor 15, ST2, high-sensitivity troponin $\mathrm{T}$, and $\mathrm{N}$-terminal pro brain natriuretic peptide in heart failure with preserved vs. reduced ejection fraction. Eur J Heart Fail 2012;14:1338-47.

15. Nagueh SF, Smiseth OA, Appleton CP, et al. Recommendations for the evaluation of left ventricular diastolic function by echocardiography: An Update from the American Society of Echocardiography and the European Association of Cardiovascular Imaging. J Am Soc Echocardiogr 2016;29:277-314.
16. Lang RM, Badano LP, Mor-Avi V, et al. Recommendations for cardiac chamber quantification by echocardiography in adults: an update from the American Society of echocardiography and the European association of cardiovascular imaging. J Am Soc Echocardiogr 2015;28:1-39.

17. Beran TN, Violato C. Structural equation modeling in medical research: a primer. BMC Res Notes 2010;3:261-7.

18. Tan ESJ, Yap J, Xu CF, et al. Association of age, sex, body size and ethnicity with electrocardiographic values in community-based older Asian adults. Heart Lung Circ 2016;25:705-11.

19. Chiladakis J, Kalogeropoulos A, Zagkli F, et al. Effect of heart rate on the intrinsic and the ventricular-paced QRS duration. J Electrocardio/ 2015;48:689-95.

20. Mason JW, Badilini F, Vaglio M, et al. A fundamental relationship between intraventricular conduction and heart rate. J Electrocardiol 2016;49:362-70.

21. Hancock EW, Deal BJ, Mirvis DM, et al. AHA/ACCF/HRS recommendations for the standardization and interpretation of the electrocardiogram: Part V: electrocardiogram changes associated with cardiac chamber hypertrophy: a scientific statement from the American Heart Association electrocardiography and arrhythmias Committee, Council on clinical cardiology; the American College of cardiology Foundation; and the heart rhythm Society. endorsed by the International Society for computerized Electrocardiology. J Am Coll Cardiol 2009;53:992-1002.

22. Otaki Y, Takahashi H, Watanabe T, et al. Electrocardiographic left ventricular hypertrophy Cornell product is a feasible predictor of cardiac prognosis in patients with chronic heart failure. Clin Res Cardiol 2014;103:275-84.

23. Martinez-Rumayor AA, de Lemos JA, Rohatgi AK, et al. Addition of highly sensitive troponin T and N-terminal pro-B-type natriuretic peptide to electrocardiography for detection of left ventricular hypertrophy: results from the Dallas heart study. Hypertension 2013;61:105-11.

24. Gerdts E, Okin PM, Boman K, et al. Association of heart failure hospitalizations with combined electrocardiography and echocardiography criteria for left ventricular hypertrophy. Am J Hypertens 2012;25:678-83.

25. Sosner P, Cabasson S, Hulin-Delmotte $C$, et al. Effect of Cornell product and other ECG left ventricular hypertrophy criteria on various cardiovascular endpoints in type 2 diabetic patients. Int J Cardiol 2014;175:193-5.

26. Ishikawa J, Ishikawa S, Kabutoya T, et al. Cornell product left ventricular hypertrophy in electrocardiogram and the risk of stroke in a general population. Hypertension 2009;53:28-34.

27. Sundström J, Lind L, Arnlöv J, et al. Echocardiographic and electrocardiographic diagnoses of left ventricular hypertrophy predict mortality independently of each other in a population of elderly men. Circulation 2001;103:2346-51.

28. Okin PM, Devereux RB, Harris KE, et al. Regression of left ventricular hypertrophy is associated with less hospitalization for heart failure in hypertensive patients. Ann Intern Med 2007;147:311-9.

29. Okin PM, Devereux RB, Jern $S$, et al. Regression of electrocardiographic left ventricular hypertrophy during antihypertensive treatment and the prediction of major cardiovascular events. JAMA 2004;292:2343-9.

30. van Veldhuisen DJ, Linssen GCM, Jaarsma T, et al. B-type natriuretic peptide and prognosis in heart failure patients with preserved and reduced ejection fraction. J Am Coll Cardiol 2013;61:1498-506. 